\title{
Investigating Wearable Technology for Fatigue Identification in the Workplace
}

\author{
Christopher Griffiths, Judy Bowen, Annika Hinze \\ The University of Waikato, New Zealand \\ cjgglestudents.waikato.ac.nz, jbowen@waikato.ac.nz, \\ hinze@waikato.ac.nz,
}

\begin{abstract}
Fatigue has been identified as a significant contributor to workplace accident rates. However, risk minimisation is a process largely based on selfreporting methodologies, which are not suitable for fatigue identification in high risk industries. Wearable technology which is capable of collecting physiological data such as step and heart rates as an individual performs workplace tasks has been proposed as a possible solution. Such devices are minimally intrusive to the individual and so can be used throughout the working day. Much is promised by the providers of such technology, but it is unclear how suitable it is for in-situ measurements in real-world work scenarios. To investigate this, we performed a series of studies designed to capture physiological and psychological data under differing (physical and mental) loading types with the intention of finding out how suitable such equipment is. Using reaction time (simple and choice) as a measure of performance we found similar correlations exist between loading duration and our measured indicators as those found in large-scale laboratory studies using state of the art equipment. Our results suggest that commercially available activity monitors are capable of collecting meaningful data in workplaces and are, therefore, worth investigating further for this purpose.
\end{abstract}

\section{Introduction}

Fatigue is, by nature, cumulative, and is influenced by many variables such as activity, time of day, sleep levels and social pressures. The impact of high fatigue levels, especially in high-risk workplaces, can lead to increased risk for employees, and is seen as a large contributor to workplace accident rates [29, 12, 19]. Risk assessment processes include attempts to assess the impact of workplace activities on fatigue levels. Typically these use qualitative self-reporting methodologies rather than quantitative data measurements. This type of data collection in workplace contexts is susceptible to response bias [24] and can result in a generalised view of risk. However the actual impact of activity upon fatigue is individualised. For example, tasks performed by a young person impacts fatigue levels to a lesser extent than tasks performed by an older person [2].

Many studies investigating the impact of activity upon performance have been undertaken. Most identify that an individual's performance is negatively impacted by activity, both physical and mental [27, 16, 23,9]. Each of these studies collected data using different methods, but the majority are conducted in a laboratory setting with large numbers of participants. In real-world work environments, individuals encounter naturally occurring stressors that may not be observed in laboratory experiments. Similarly 
the longer-term nature of fatiguing activities in the workplace and the limited number of participants who can be measured in in-situ studies may make it hard to reproduce known results.

The rise in popularity of wearable devices has provided additional tools for quantification of individualised activity levels. These devices are designed to operate autonomously and enable the real-time collection of quantifiable data in the field throughout the day. Physiological markers such as step and heart rates can be used to determine workload intensities, especially in physically-biased roles. Conversely, changes in the balance of the autonomic nervous system can be used to quantify cognitive loading. However, it is unclear how accurate these measures are when using commercial products designed primarily for the personal user. As a first investigation into this, we performed a series of single-person studies designed to see if we could reproduce known correlations between activity (both mental and physical) and response times. If we find similar correlations can be identified by these devices then it is worth further investigating their use in real-world work environments.

In this paper, we present the results of these initial studies investigating the use of low cost commercially available devices as a means of collecting data that has sufficient accuracy and granularity to identify physiological changes associated with mental and physical activity types which may indicate fatigue. We examine the suitability of such devices for use in the field, specifically for gathering data and monitoring of forestry workers throughout their working day.

\subsection{Motivation}

The forestry industry in New Zealand has a poor Health and Safety record with some 12,921 active Accident Compensation Corporation (ACC) claims between 2008 and 2013. More importantly the number of reported fatalities for the same period is 32 . Such high levels of fatalities are concerning to the industry with reforms being planned to address safety of employees. Suggestions based on experimental data are limited, and difficult to source. Currently, the only practicable solution is seen as increasing the level of mechanisation resulting in removal of the employee from the worksite.

Recently (2014), the large numbers of incidents reported prompted the Independent Forestry Safety Review [1], designed to investigate factors that impact on health and safety within the forestry industry and to provide guidelines designed to minimize the number of incidents. It was identified that the forestry industry is one of the most dangerous occupations in New Zealand. Employees are 15 times more likely to suffer a workplace injury compared to other NZ based industries.

The physical nature of the work, long working days and tasks requiring high levels of concentration can all exacerbate the impact of fatigue [13,28] with high demand tasks requiring more energy to complete. In workplaces with high task demands employees may experience the effects of fatigue earlier. There is also a recognition that the lack of welfare facilities in the forestry environment may be an additional contributor to fatigue. 


\subsection{Measurements}

There are numerous physiological indicators that can be used to quantify activity. Step and heart rates have been successfully used to quantify physical activity [8], whereas changes in the balance of the autonomic nervous system have been used as an indicator of cognitive activity [25].

Currently seen as the gold standard for measurement of these variables are step counting for ambulatory activity and, electrocardiography for heart rate data collection. However, we must remember that in our proposed domain the devices we choose need to be minimally intrusive and capture data autonomously. Before we can assess suitable devices for the data collection we must first consider what the appropriate data to collect is. We discuss proposed identifiers of activity next, these will then inform subsequent choices of appropriate apparatus for data capture.

Step rate: The use of pedometers to measure an individual's daily activity has been used many times in differing domains. Designed to capture accelerations of the hip during gait cycles they count the number of steps taken by an individual over a given time period. Using the number of steps taken we can gain an insight into how role-based activity may contribute to excessive fatigue levels. This method has been used successfully to differentiate activity levels by role types in forestry harvesting operations [17] with large differences between manual and mechanised activity types being identified.

Heart Rate: Heart rate data captured throughout the course of a work period can be an used as an indicator of task demand. Higher heart rates typically accompany higher periods of physical activity as higher levels of oxygenated blood are required due to increased physiological demand. Increases in heart rate are individualized with demand being dependent on such criteria as age and fitness levels. However, maximal heart rate can be calculated from the general formula equation Maximal Heart Rate $=220$ minus Age [20]. The resultant figure can then be used in conjunction with resting heart rate to determine periods of high and low activity where activity is defined as deviation from the resting heart rate.

Heart rate variability (HRV): Heart rate variability is the time interval between successive heart beats. Shorter periods are indicative of active loading while longer time periods are indicative of rest. There are a large number of variables that can be extracted from collected data each providing an insight into autonomic nervous system activity. For our studies we use the low frequency/high frequency ratio of the power spectrum density of the heart. This variable has been found to correlate well with mental activity across differing levels of cognitive load [7, 10,26].

Workload intensity: Workload intensity, or how hard an individual perceives their workload can be an indicator of increasing fatigue levels. Monotonous or repetitive tasks have been identified as a contributor to motivational levels [22] with individual performance slowing as motivational levels decrease [34]. The Task Load Intensity Tool (TLX) developed at the Ames Research Center is designed as a self-reporting estimator of how difficult an individual perceives their workload. Using a six point scale it provides a workload score for workplace activities [18]. Increasing workload scores for tasks where the only change is duration can indicate increasing workplace fatigue [3]. 
Performance: Reaction time (both simple and choice) has been used many times to measure individual performance. It is a measure of the elapsed time between the presentation of a given stimulus and the participant's response. In simple reaction time the user responds to one stimulus whereas in choice reaction time the user must identify the correct response from a set of choices. Reaction time has been found to deteriorate with increasing fatigue $[13,5,21]$ furthermore the time difference between choice and simple reaction time can give insights into the speed of mental processing.

\section{State of the Art Methods and Tools}

When considering our methodologies for collecting data we must also consider the accuracy of our proposed devices. The most accurate measure for step counting is manual counting. However, this is impractical for many purposes and in particular for our chosen domain - one cannot manually count steps in the workplace. The Yamax range of pedometers are widely regarded as the most accurate equipment for automatic step counting [32] and have been used in many studies investigating step rates [8,6,30]. When comparing the accuracy of our chosen device (Fitbit Charge HR) many studies have found good agreement between actual and recorded steps taken $[14,15]$.

Real-time data collection of cardiovascular activity in the workplace presents additional challenges. This type of data collection is typically done using an electrocardiograph and is undertaken at hospitals or dedicated research laboratories. The equipment can be bulky and cumbersome with an individual having wired sensors placed on the body. As previously discussed, our proposed domain is forestry operations in which traditional ECG measurement is not practical.

Wearable devices exist that are designed to capture data in free living activities. These devices range from chest strap based monitoring through to smart clothing containing electrodes to capture the electrical signals produced during a heart beat cycle. Devices are designed to be minimally intrusive to the individual and collect data autonomously facilitating use in the field.

The Polar range of products have been used extensively in studies investigating the cardiovascular system. For example, Paritala's work investigating the effects of physical and mental activity used the Polar RS800 monitor to capture the heart rate of 24 participants during laboratory testing [25]. In a similar study the relationship between markers of work related fatigue and HRV of 28 participants used Polar devices for the data capture [33].

In the above section we have discussed the gold standards for data collection and identified limitations for use in workplace domains. Tools for performance and perceived workload intensity are numerous however, given our proposed domain our choice is limited. Any devices used to measure our required metrics will be worn throughout the working day by forestry workers and so should not cause discomfort during their physical activities. In order to collect data we therefore selected to use Fitbit Charge HR activity trackers to collect ambulatory data and the Polar RS8020CX fitness watch paired with a Polar chest strap to collect heart-rate data. In addition, field-based testing must be quick to conduct to minimise the impact on both productivity and the individual and as such we implemented an electronic version of the unweighted NASA TLX to 
collect data on perceived workload intensity as this will be quicker than a paper-based survey. For performance data we selected the Deary-Liewald Reaction Time application developed by the Centre for Cognitive Ageing and Cognitive Epidemiology [11]. It is an application specifically designed for conducting reaction time testing using portable computing devices.

\section{Studies}

The aim of these initial studies was to investigate whether the tracking devices and tools listed above could be used to replicate the results of large-scale, laboratory-based tests which investigate the effects of activity on fatigue. Performing monitoring studies of forestry workers is time-consuming and requires considerable buy-in from a number of different entities (forestry owners, contracting companies, health and safety organisations and the workers themselves). Before undertaking the field studies with workers, we therefore wanted to be certain that our equipment choices would be suitable and we could gather meaningful data. As such we focussed on evaluating combinations of different measurements across different activity types. Each of the studies described below were conducted with a single participant over short periods of time (the course of a day or a focussed activity) as a means to conduct such an evaluation.

\subsection{Activity and Recovery}

To assess the impact of activity on the physiological and psychological systems our first study was designed to assess the impact of differing loading types (physical and mental) on performance and psychological indicators. During physical loading the measurements were undertaken in an ad-hoc manner as and when opportunities presented themselves. Workload intensity was measured using an electronic implementation of the NASA TLX running on a dedicated server and measured at the same time as reaction time testing. Apparatus was worn for the duration of the monitoring exercise.

\subsection{Physical loading}

Monitoring sessions were undertaken over the course of a working day. The participant worked as a floor team member at a large retail outlet, a position requiring large amounts of ambulatory activity. Both ambulatory activity and heart rate data collection commenced at the start of the work day and concluded at the end of the work period. Workload intensity and performance measurement was undertaken at the start and end of the work period and at the participant's designated break times $(2 \times 15$ mins and $1 \mathrm{X}$ 30mins).

When extracting data for heart rate variability, the raw data is put through an analysis program that computes the various indicators of cardiographic activity. For this task we used the gHRV software developed by Milegroup based at the University of Vigo in Spain. The application is specifically designed for the analysis of heart rate variability. ${ }^{1}$

\footnotetext{
${ }^{1}$ available from https://milegroup.github.io/ghrv/doc.html
} 
As mentioned in 1.2, we use the ratio of low frequency to high frequency of the power spectrum density of the heart as an indicator of autonomic nervous system activity, inferring increasing fatigue from the increase of this ratio. The large datasets we create during monitoring can be used to provide point data, however, for our estimation of increasing fatigue we use the cumulative mean of the data over the duration of the monitoring period.

When assessing performance as the speed of mental processing (choice reaction time minus simple reaction time) we see an initial period of improvement followed by a period of slowing as the workday lengthens. The psychological impact was found to increase across duration with increasing perceived task intensity being reported. (Figure 1)
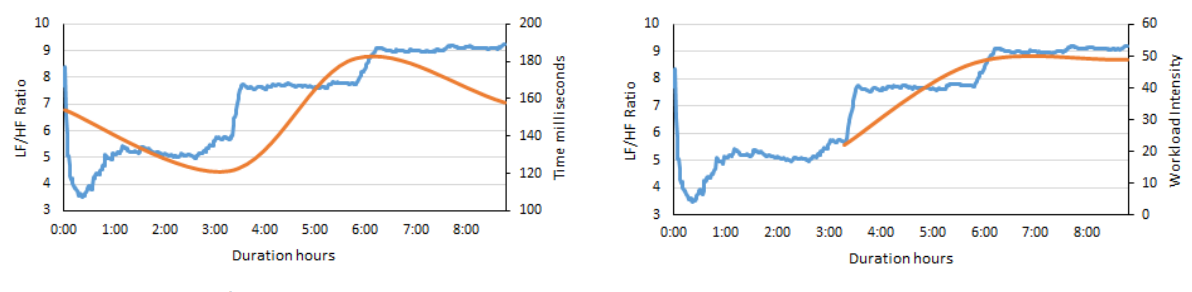

Fig. 1: Speed of mental processing (left) and workload intensity (right) vs cumulative mean $\mathrm{LF} / \mathrm{HF}$ ratio

Activity during this period was measured as 22,322 steps $(18.8 \mathrm{~km})$ with the majority occurring within the first 6 hours of the work session. The graphs indicate that speed of mental processing and perceived workload intensity are impacted by this high physical loading.

\subsection{Cognitive loading}

To investigate the impact of cognitive load we conducted experiments where driving was used as the mental activity and stressor. Driving is a task requiring constant vigilance and high levels of spatial awareness. The experimenter performed a 3 hour driving exercise on real roads encountering typical driving conditions. Reaction time testing in conjunction with workload intensity monitoring was undertaken at 30 minute intervals for the duration of the experiment. On completion of the driving exercise monitoring was continued to assess performance through a recovery period.

We found a similar increasing trend as that in physical loading however, the increase in $\mathrm{LF} / \mathrm{HF}$ ratio was found to be higher.Workload intensity and performance were also negatively impacted over the duration of the experiment (Figure 2).

The higher number of data points collected during the driving experiments facilitates further analysis of existing relationships between our variables. We identified a good correlation for the majority of our measured variables. Table 1 presents the results of the correlation between our measured variables. 


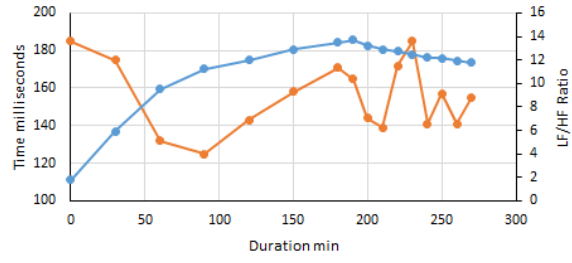

$\rightarrow$ Speed of mental processing $\rightarrow$ Cumulative mean LF/HF Ratio

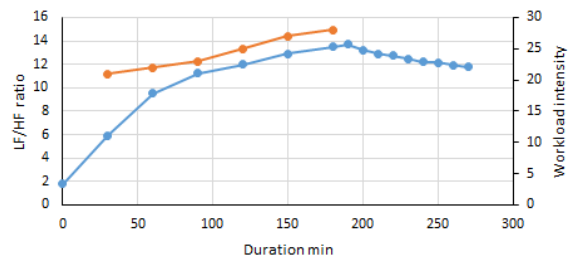

$\rightarrow$ LF/HF Ratio $\rightarrow-T L$

Fig. 2: Speed of mental processing (left) and workload intensity (right) v cumulative mean $\mathrm{LF} / \mathrm{HF}$ ratio for driving experiment

Table 1: Correlation of physiological and psychological variables

\begin{tabular}{|c|c|l|}
\hline Condition & Correlation R & Comments \\
\hline \hline Driving v Speed of Mental Processing & 0.73 & Speed of Mental Processing increases with loading duration \\
\hline Driving v Workload Intensity & 0.98 & Perceived workload increases with loading duration \\
\hline Driving v LF/HF Ratio & 0.89 & LF/HF Ratio increases with loading duration \\
\hline Recovery v Speed of Mental Processing & -0.69 & Speed of Mental Processing decreases on loading cessation \\
\hline Recovery v LF/HF Ratio & -0.75 & LF/HF Ratio decreases on cessation of loading \\
\hline LF/HF Ratio v Workload Intensity & 0.92 & LF/HF Ratio increases with increasing workload perception \\
\hline LF/HF Ratio v Speed of Mental Processing driving & 0.72 & Speed of Mental Processing increases with increasing LF/HF Ratio \\
\hline Speed of Mental Processing v Workload Intensity & 0.56 & Speed of Mental Processing increases with increasing perceived workload \\
\hline
\end{tabular}

\section{Discussion}

When examining the results of our studies, we can see that each of our measured variables indicate an impact resulting from activity. In both physical and cognitive loading types, we see a reduction in performance across loading duration indicated by slowing of reaction times. We also observe that this reduction in performance occurs as the cumulative mean of low frequency/high frequency ratio increases (Figure 1). Furthermore, we note that cognitive load impacts the cumulative mean of low frequency/high frequency ratio to a greater extent than physical loading with higher values being recorded (Figure 2) for our driving study. These results are similar to the findings of other studies investigating the impact mental and physical loading on the autonomic nervous system $[27,10,23]$.

In our driving study, we found a good correlation between our measured variables (Table 1) indicating relationships between performance and increasing levels of the high frequency/low frequency ratio. We also identified that performance continues to degrade on cessation of activity prior to improvement facilitated through a recovery period. These findings agree with those of previous studies investigating the impact of loading on the autonomic nervous system [10,9].

Similar to other studies [9], we found perceived workload intensity increases as loading duration lengthens, individuals report higher workload scores later in the day for the same tasks. The perception of workload intensity was also found to relate to our 
measured physiological indicators. We identified higher workload scores were reported with increasing cumulative mean low frequency/high frequency ratios.

What we are seeking to identify here is the suitability of wearable devices for capturing meaningful data. As such the results described are not intended to prove relationships between activity, fatigue and response times per se. but rather act as a proof of concept that such methods and tools can replicate known correlations in such data. Given that they indicate this is possible, we can then move on to study their use in the field within our larger-scale studies with forestry workers.

\section{Conclusions and Future Work}

Our research question asked if low cost lightweight methods can be used for meaningful data collection of data pertaining to physiological indicators related to the impact of activity on performance. As found in other studies [31,4] the Fitbit Charge HR successfully collected data on ambulatory activity. Having a similar footprint to a wrist watch we found the device minimally intrusive, and caused no discomfort to the individual.

The Polar chest strap used for the collection of heart rate variability proved to be capable of collecting meaningful data. However, the practicality of this device can be called into question. We found the fastening clasp on the chest strap can cause discomfort to the individual, resulting in reluctance to wear the device over longer periods. We also note a period of spiking may be present due to insufficient moisture between skin and electrode to accurately record heart rate data. This can be overcome by the application of electrode gel prior to the commencement of data capture.

As in other studies [29, 3, 16], our results indicate workplace activity impacts both physiological and psychological states. We identify increasing values of the cumulative mean low frequency/high frequency ratio as loading duration extends. Furthermore, we identify that cognitive loading has a greater impact on the individual than physical loading. We have also identified that on cessation of activity performance continues to degrade prior to improvement. We acknowledge that monitoring undertaken during our study was brief and extended data collection over longer time frames is required to further identify any trends that maybe present. Furthermore, we acknowledge that the data collected represents a single individual as such future studies should incorporate a larger participant base to gain a better understanding of the physiological and psychological impact of workplace activity.

In conclusion, we assessed commercially available fitness monitoring devices as tools for physiological data capture under differing loading types. We found that the selected activity trackers are capable of collecting meaningful data providing researchers with additional tools for monitoring activity in free living. The use of the cumulative mean of the power spectrum density of the heart may be a useful indicator of the impact of activity on the autonomic nervous system and as such may be useful for the determination of workplace role/task intensity. 


\section{References}

1. Adams, G., Armstrong, H., Cosman, M.: Independent forestry safety review. Final Report (2014)

2. Avin, K.G., Law, L.A.F.: Age-related differences in muscle fatigue vary by contraction type: a meta-analysis. Physical therapy 91(8), 1153 (2011)

3. Baulk, S.D., Kandelaars, K.J., Lamond, N., Roach, G.D., Dawson, D., Fletcher, A.: Does variation in workload affect fatigue in a regular 12-hour shift system? Sleep and Biological Rhythms 5(1), 74-77 (2007)

4. Bowen, J., Hinze, A., Cunningham, S.J., Parker, R.: Investigating the use of activity trackers to observe high-risk work environments (2015)

5. Brisswalter, J., Arcelin, R., Audiffren, M., Delignieres, D.: Influence of physical exercise on simple reaction time: effect of physical fitness. Perceptual and motor skills 85(3), 1019-1027 (1997)

6. Castillo-Retamal, M., Hinckson, E.A.: Measuring physical activity and sedentary behaviour at work: a review. Work 40(4), 345-357 (2011)

7. Chandola, T., Britton, A., Brunner, E., Hemingway, H., Malik, M., Kumari, M., Badrick, E., Kivimaki, M., Marmot, M.: Work stress and coronary heart disease: what are the mechanisms? European heart journal 29(5), 640-648 (2008)

8. Choi, S.W., Lee, J.H., Jang, Y.K., Kim, J.R.: Assessment of ambulatory activity in the republic of korea navy submarine crew. Undersea \& Hyperbaric Medicine 37(6), 413 (2010)

9. Cinaz, B., La Marca, R., Arnrich, B., Tröster, G.: Monitoring of mental workload levels. In: Proceedings of IADIS eHealth conference. pp. 189-193 (2010)

10. Collet, C., Averty, P., Dittmar, A.: Autonomic nervous system and subjective ratings of strain in air-traffic control. Applied ergonomics 40(1), 23-32 (2009)

11. Deary, I.J., Liewald, D., Nissan, J.: A free, easy-to-use, computer-based simple and fourchoice reaction time programme: the deary-liewald reaction time task. Behavior Research Methods 43(1), 258-268 (2011)

12. Dobbie, K.: Fatigue-related crashes: An analysis of fatigue-related crashes on Australian roads using an operational definition of fatigue. No. OR 23 (2002)

13. Ellis, H.D.: The effects of cold on the performance of serial choice reaction time and various discrete tasks. Human Factors 24(5), 589-598 (1982)

14. Evenson, K.R., Goto, M.M., Furberg, R.D.: Systematic review of the validity and reliability of consumer-wearable activity trackers. International Journal of Behavioral Nutrition and Physical Activity 12(1), 159 (2015)

15. Ferguson, T., Rowlands, A.V., Olds, T., Maher, C.: The validity of consumer-level, activity monitors in healthy adults worn in free-living conditions: a cross-sectional study. International Journal of Behavioral Nutrition and Physical Activity 12(1), 42 (2015)

16. Galy, E., Cariou, M., Mélan, C.: What is the relationship between mental workload factors and cognitive load types? International Journal of Psychophysiology 83(3), 269-275 (2012)

17. Griffiths, C.J.G.: Investigating Tools and Methods for Data Capture of Forestry Workers. Ph.D. thesis, University of Waikato (2016)

18. Hart, S.G., Staveland, L.E.: Development of nasa-tlx (task load index): Results of empirical and theoretical research. Advances in psychology 52, 139-183 (1988)

19. Haslam, R.A., Hide, S.A., Gibb, A.G., Gyi, D.E., Pavitt, T., Atkinson, S., Duff, A.: Contributing factors in construction accidents. Applied ergonomics 36(4), 401-415 (2005)

20. Karvonen, M.J., Kentala, E., Mustala, O.: The effects of training on heart rate; a longitudinal study. In: Annales medicinae experimentalis et biologiae Fenniae. vol. 35, p. 307 (1957)

21. Lim, J., Dinges, D.F.: Sleep deprivation and vigilant attention. Annals of the New York Academy of Sciences 1129(1), 305-322 (2008) 
22. MacDonald, W.: The impact of job demands and workload on stress and fatigue. Australian Psychologist 38(2), 102-117 (2003)

23. McDuff, D., Gontarek, S., Picard, R.: Remote measurement of cognitive stress via heart rate variability. In: Engineering in Medicine and Biology Society (EMBC), 2014 36th Annual International Conference of the IEEE. pp. 2957-2960. IEEE (2014)

24. Morrel-Samuels, P.: Getting the truth into workplace surveys. Harvard business review 80(2), 111-118 (2002)

25. Paritala, S.A.: Effects of physical and mental tasks on heart rate variability. Ph.D. thesis, Kakatiya University, India (2009)

26. Patel, M., Lal, S., Kavanagh, D., Rossiter, P.: Applying neural network analysis on heart rate variability data to assess driver fatigue. Expert systems with Applications 38(6), 7235-7242 (2011)

27. Pichot, V., Bourin, E., Roche, F., Garet, M., Gaspoz, J.M., Duverney, D., Antoniadis, A., Lacour, J.R., Barthélémy, J.C.: Quantification of cumulated physical fatigue at the workplace. Pflügers Archiv 445(2), 267-272 (2002)

28. Pilcher, J.J., Nadler, E., Busch, C.: Effects of hot and cold temperature exposure on performance: a meta-analytic review. Ergonomics 45(10), 682-698 (2002)

29. Rosa, R.R.: Extended workshifts and excessive fatigue. Journal of sleep research 4(s2), 5156 (1995)

30. Soroush, A.: A 6 month physical activity intervention in university staff: effectiveness and health outcomes-The ASUKI Step Study. Inst för biovetenskaper och näringslära/Dept of Biosciences and Nutrition (2013)

31. Takacs, J., Pollock, C.L., Guenther, J.R., Bahar, M., Napier, C., Hunt, M.A.: Validation of the fitbit one activity monitor device during treadmill walking. Journal of Science and Medicine in Sport 17(5), 496-500 (2014)

32. Tully, M.A., McBride, C., Heron, L., Hunter, R.F.: The validation of fitbit zip physical activity monitor as a measure of free-living physical activity. BMC research notes 7(1), 952 (2014)

33. Völker, I., Kirchner, C., Bock, O.L.: Relation between multiple markers of work-related fatigue. Safety and Health at Work 7(2), 124-129 (2016)

34. Wright, R.A., Pantaleo, G.: Effort processes in achieving performance outcomes: interrelations among and roles of core constructs. Behavioral and Brain Sciences 36(06), 705-706 (2013) 